\title{
Successful Management of a Rare Complication After Percutaneous Native Renal Biopsy
}

\author{
Yasser W. Al-Mula Abed ${ }^{\mathrm{a}, \mathrm{c}}$, Rana S. Hamid ${ }^{\mathrm{b}}$, Anupam K. Kakaria ${ }^{\mathrm{b}}$, \\ Saif A. Khan ${ }^{a}$, Saja M. Mohammed ${ }^{\text {a }}$
}

\begin{abstract}
Percutaneous renal biopsy is presumed to be a relatively safe procedure that is routinely performed to help in the diagnosis, prognosis and treatment of various renal parenchymal diseases. It is widely performed by interventional nephrologists and radiologists for certain indications. Here, we report a young man who developed a significant retroperitoneal hematoma and active bleeding due to left 12th intercostal artery injury after a native renal biopsy, with a concomitant renal segmental artery pseudoaneurysm. The bleeding was successfully treated by selective transcatheter arterial embolization with coils.
\end{abstract}

Keywords: Hematoma; Renal biopsy; Renal artery; Pseudoaneurysm; Bleeding; Intercostal artery; Embolization

\section{Introduction}

Percutaneous renal biopsy is an essential tool in the diagnosis and treatment of patients with renal diseases. It was first introduced by Iversen and Brun in 1951 [1]. Radiological localization and ultrasonographic guidance has made the procedure simpler and more accurate with less morbidity [2]. The most common complication of percutaneous renal biopsy is bleeding which may present as hematuria, intrarenal or perirenal hematoma, or any combination of these. Renal or extrarenal bleeding could be serious and life-threatening. The reported incidence of bleeding following the procedure varies widely depending on the type of method used for detection. A prospective study has shown that a perinephric hematoma can be found in $90 \%$ of patients after renal biopsy, out of which only

Manuscript accepted for publication September 25, 2014

aDepartment of Medicine, Sultan Qaboos University Hospital, Muscat, Sultanate of Oman

${ }^{b}$ Department of Radiology and Molecular Imaging, Sultan Qaboos University Hospital, Muscat, Sultanate of Oman

${ }^{\mathrm{c} C}$ Corresponding Author: Yasser W. Al-Mula Abed, Nephrology Unit, Department of Medicine, Sultan Qaboos University Hospital, PO Box 35, AlKhodh 123, Muscat, Sultanate of Oman. Email: mulaabed@yahoo.com

doi: http://dx.doi.org/10.14740/jmc1963w
$6 \%$ were clinically significant [3]. Proper detection of complication by close observation, serial abdominal examination and serial hemoglobin level measurement followed by proper imaging, if suspected, is crucial.

\section{Case Report}

A 21-year-old man underwent a percutaneous left kidney biopsy. The biopsy was done as part of evaluation of unexplained advanced renal failure that was discovered in this patient about 2 months prior to his admission and required hemodialysis in a local hospital. His plasma creatinine was more than 1,500 $\mu \mathrm{mol} / \mathrm{L}$ before hemodialysis (and $812 \mu \mathrm{mol} / \mathrm{L}$ after hemodialysis). He had nephrotic range proteinuria and microscopic hematuria with red cell casts and negative immunological workup. His coagulation profile and platelets count were normal. The biopsy was done using the $18-\mathrm{G}$ biopsy needle with guidance by an ultrasound. The procedure was uneventful without any immediate complications. The biopsy showed features of end-stage renal disease with mesangioproliferative glomerulonephritis suggestive of advance IgA nephropathy.

Few hours after biopsy, the patient complained of abdominal pain and his hemoglobin dropped from 9 to $6.8 \mathrm{~g} / \mathrm{dL}$. An urgent ultrasound study proved the presence of a small retroperitoneal hematoma. He was supported with blood transfusion but the next day he developed peritonism and became anuric. An urgent CT angiogram of abdomen (plain, arterial and portovenous phases) showed a large left retroperitoneal hematoma (measuring $7 \times 10 \times 18 \mathrm{~cm}$ ) extending from the subsplenic region down to left iliac fossa causing displacement of the adjacent organs. There was active contrast extravasation in the posterior abdominal wall in the arterial phase suggesting arterial bleed. There was also a tiny area of abnormal arterial blush in the left renal parenchyma suspicious for bleed from a left renal segmental pseudoaneurysm with perirenal hematoma at inferior pole (Fig. 1).

A multi-disciplinary discussion was held with a decision to proceed with angiography and possible embolization. Selective left renal angiogram was performed and proved evidence of a subcentimetric pseudoaneurysm filling from the intrarenal branches from the lower pole left renal artery. There was no active bleed from this pseudoaneurysm. Superselective catheterization using a micro-catheter was performed for the ar- 


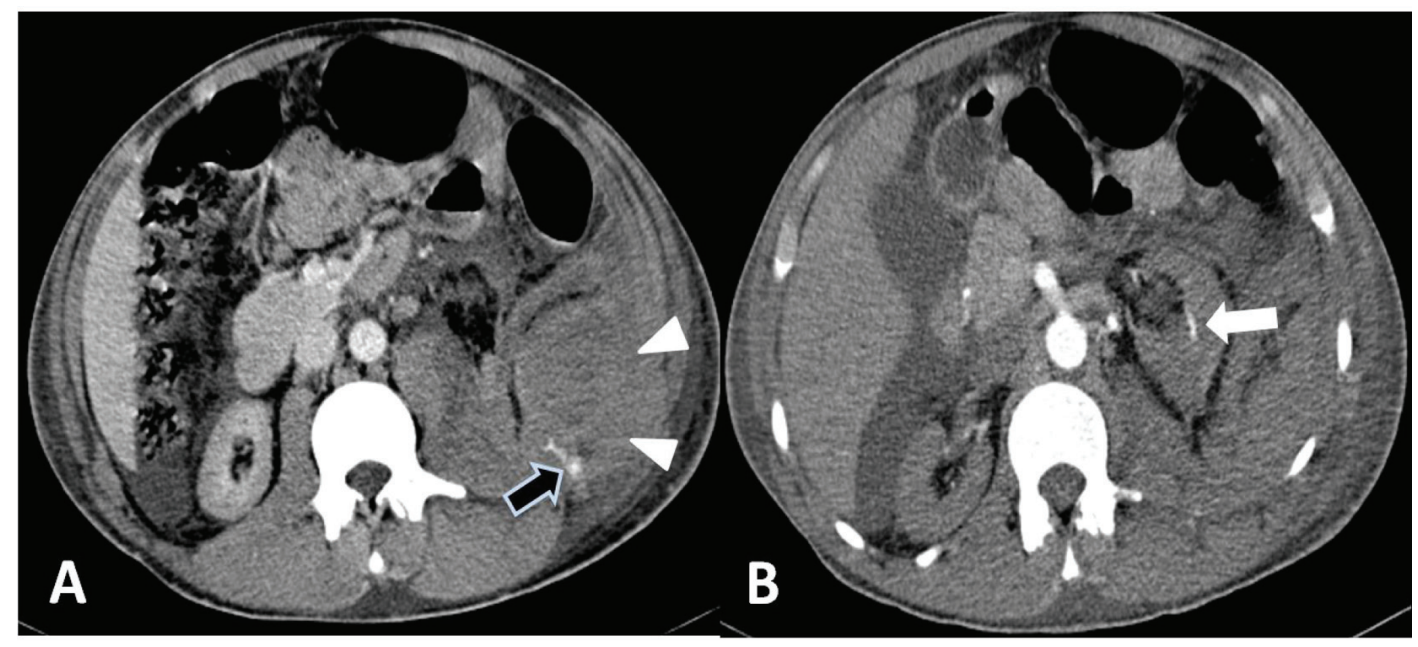

Figure 1. Axial CT scan of abdomen in arterial phase. There is a left lumbar retroperitoneal hematoma (arrowheads) displacing the kidney anteriorly. A small area of contrast extravasation is seen in posterior abdominal wall suggesting active bleed (black arrow). A tiny area of abnormal arterial blush is also seen in left renal parenchyma suspicious for bleed (white arrow).

tery supplying the pseudoaneurysm. The feeding artery was occluded using three $3 \mathrm{~mm} \times 4 \mathrm{~cm}$ platinum coils. Subsequent angiogram of the left 12 th intercostal artery revealed active extravasation of contrast from this artery distally. Selective catheterization using a micro-catheter was performed and this artery was then occluded distally using three $2 \mathrm{~mm} \times 2 \mathrm{~cm}$ coils with excellent result (Fig. 2).

The patient remained hemodynamically stable with no further drop in hemoglobin level. The abdominal pain gradually ceased and he was discharged in a stable condition $48 \mathrm{~h}$ after the procedure with an advice to continue regular hemodialysis on an outpatient basis. Subsequent feedback from the local hospital confirmed that he is stable with no further requirement of blood transfusion. Transplant workup is planned to be done soon.

\section{Discussion}

Renal hematomas are the most commonly reported complications after percutaneous renal biopsies but fortunately these are self-limited in most of cases with no need for active intervention [4]. Other complications like arteriovenous fistula, colonic injury, infections or pneumothorax are much less frequent lifethreatening, and stated to occur in less than $0.1 \%$ of cases [5]. Renal artery pseudoaneurysm is another uncommon complication that could be fatal in case ruptured [6]. The prevalence has significantly reduced recently mainly due to the widespread use of ultrasound guidance and automated-gun biopsy devices [7]. These complications are less common in transplanted than in native kidneys biopsies [8].

The persistence of abdominal or loin pain, gross hematuria, development of oliguria or anuria and hemodynamic instability, like hypotension and tachycardia, are clinical indicators of a significant bleed after renal biopsy. Ultrasound should be done at the minimal suspicion to rule out the presence of subcapsular or perirenal hematoma. This should be followed by
CT study which can further clarify any abnormal ultrasound finding. Active bleeding can be identified with the use of contrast enhancement and further actions can be decided according to the severity [9]. However, false-negative results can be obtained if the bleeding is less than $0.5 \mathrm{~mL} / \mathrm{min}$ [10].

Endovascular embolization is a very effective method for managing patients who do not respond to conservative treatment. When compared to surgery, it is relatively simpler, more safe, and gives prompt results in controlling the hemorrhage and it is life saving [11]. Although metallic pushable coils are the most frequently used embolic agents, however other embolic agents like microparticles, gelfoam and rarely use of glue has also been described in literature [12-14].

The international literature is replete with case reports on arterial embolization after complicated renal biopsy but our case is rare as two different vessels were injured by single biopsy path and both were successfully treated by coil embolization.

\section{Conclusion}

Hemorrhage post-renal biopsy is usually self-limited and rarely requires intervention. Massive hemorrhage causing hemodynamic instability is a rare complication and usually arises from renal artery. Injuries of extrarenal arteries by renal biopsy needle are very rare complications of the procedure and must always be kept in mind especially in obese patients. It may represent a diagnostic and therapeutic dilemma. Selective transcatheter arterial embolization is increasingly considered as an acceptable, minimally invasive and reliable method of managing massive acute hemorrhage which should be attempted prior to any surgical revision. However, it must be done only by experienced interventionists to avoid complications. After biopsy, an observation time of up to $24 \mathrm{~h}$ remains optimal as an observation period of less than $8 \mathrm{~h}$ risks missing more than $33 \%$ of complications. 

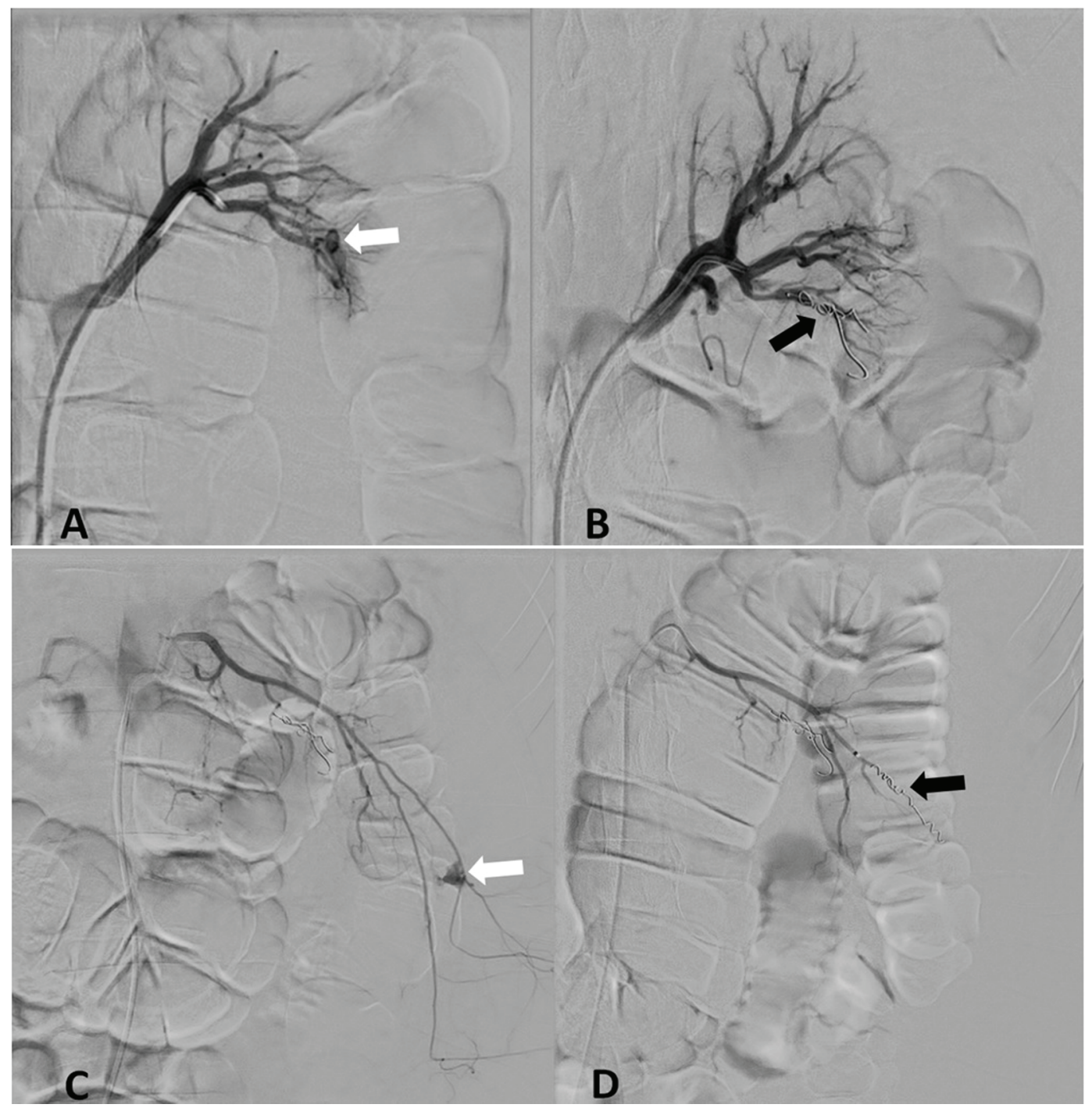

Figure 2. Digital subtraction angiogram of left renal artery (A, B). There is a small non-bleeding pseudoaneurysm in a lower pole branch of renal artery (white arrow). This was embolized with coils (black arrow). Superselective digital subtraction angiogram of left 12th intercostal artery (C, D). There is an active bleed from the artery (white arrow) which was successfully embolized with coils (black arrow).

\section{References}

1. Iversen P, Brun C. Aspiration biopsy of the kidney. Am J Med. 1951;11(3):324-330.

2. Hergesell O, Felten H, Andrassy K, Kuhn K, Ritz E. Safety of ultrasound-guided percutaneous renal biopsyretrospective analysis of 1090 consecutive cases. Nephrol Dial Transplant. 1998;13(4):975-977.

3. Whittier WL, Korbet SM. Timing of complications in percutaneous renal biopsy. J Am Soc Nephrol. 2004;15(1):142-147.

4. Lefaucheur C, Nochy D, Bariety J. [Renal biopsy: procedures, contraindications, complications]. Nephrol Ther. 2009;5(4):331-339.

5. Uppot RN, Harisinghani MG, Gervais DA. Imagingguided percutaneous renal biopsy: rationale and approach. AJR Am J Roentgenol. 2010;194(6):1443-1449.
6. Yang HK, Koh ES, Shin SJ, Chung S. Incidental renal artery pseudoaneurysm after percutaneous native renal biopsy. BMJ Case Rep. 2013;2013.

7. Sinha MD, Lewis MA, Bradbury MG, Webb NJ. Percutaneous real-time ultrasound-guided renal biopsy by automated biopsy gun in children: safety and complications. J Nephrol. 2006;19(1):41-44.

8. Herrero JC, Morales E, Carreno A, Dominguez-Gil B, Gonzalez E, Ortiz M, Hernandez E. Severe haemorrhage and retroperitoneal haematoma secondary to renal biopsy. Nephrol Dial Transplant. 2001;16(5):1078-1079.

9. Jain V, Ganpule A, Vyas J, Muthu V, Sabnis RB, Rajapurkar MM, Desai MR. Management of non-neoplastic renal hemorrhage by transarterial embolization. Urology. 2009;74(3):522-526.

10. Mavili E, Donmez H, Ozcan N, Sipahioglu M, Demirtas 
A. Transarterial embolization for renal arterial bleeding. Diagn Interv Radiol. 2009;15(2):143-147.

11. Akpinar E, Peynircioglu B, Turkbey B, Cil BE, Balkanci F. Endovascular management of life-threatening retroperitoneal bleeding. ANZ J Surg. 2008;78(8):683-687.

12. Sofocleous CT, Hinrichs CR, Hubbi B, Doddakashi S, Bahramipour P, Schubert J. Embolization of isolated lumbar artery injuries in trauma patients. Cardiovasc Intervent Radiol. 2005;28(6):730-735.
13. Geldof K, Maleux G, Heye S, Van Cleynenbreugel B, Oyen R. Transcatheter embolization of an isolated lumbar arterial bleeding complicating radical nephrectomy for renal infarction with infected perirenal haematoma. JBR-BTR. 2008;91(5):203-205.

14. Hamid RS, ul HT, Chishti I, Azeemuddin M. Post traumatic avulsion of lumbar artery: a rare cause of retroperitoneal haemorrhage treated by glue embolization. J Pak Med Assoc. 2010;60(6):487-489. 